\title{
An Efficient Magnetron Transmitter for Superconducting Accelerators
}

\author{
G. Kazakevich ${ }^{\mathrm{a}^{*}}$, V. Lebedev ${ }^{\mathrm{b}}$, V. Yakovlev ${ }^{\mathrm{b}}$, V. Pavlov ${ }^{\mathrm{c}}$ \\ ${ }^{a}$ Muons, Inc., Batavia, 65010 IL, USA, \\ ${ }^{b}$ Fermi National Accelerator Laboratory, Batavia, 65010 IL, USA, \\ ${ }^{c}$ Budker Institute of Nuclear Physics (BINP), Novosibirsk, 630090 Russia
}

\begin{abstract}
A concept of a highly-efficient high-power magnetron transmitter allowing wide-band phase and the mid-frequency power control at the frequency of the locking signal is proposed. The proposal is aimed for powering Superconducting RF (SRF) cavities of intensity-frontier accelerators. The transmitter is intended to operate with phase and amplitude control feedback loops allowing suppression of microphonics and beam loading in the SRF cavities. The concept utilizes injection-locked magnetrons controlled in phase by the locking signal supplied by a feedback system. The injection-locking signal pre-excites the magnetron and allows its operation below the critical voltage in free run. This realizes control of the magnetron power in an extended range (up to $10 \mathrm{~dB}$ ) by control of the magnetron current. Experimental studies were carried out with $2.45 \mathrm{GHz}, 1 \mathrm{~kW}, \mathrm{CW}$ magnetrons. They demonstrated stable operation of the magnetrons and the required range of power control at a low noise level. An analysis of the kinetics of the drifting charge within the framework of the presented model of phase focusing in magnetrons substantiates the concept and the experimental results.
\end{abstract}

PACS codes: 29.20.Ej, 84.40.Fe, 84.70.+p

Keywords: Magnetron, Transmitter, Superconducting accelerator, Drift equation, Pre-excited magnetron, Threshold of self-excitation

\section{Introduction}

Modern high-intensity superconducting accelerators need RF sources with an average power of tens to hundreds of kilowatts capable of supporting the phase and amplitude instability of the SRF cavity accelerating field to much less than about $1 \mathrm{deg}$. and $1 \%$, respectively. Typically compensation of the harmful effects of microphonics and beam loading is provided by the phase and power feedbacks which support accelerating field stability at the required level. Successful implementation of such feedbacks requires sufficiently wide bandwidth of the RF transmitter.

The traditional RF sources such as klystrons, Inductive Output Tubes (IOTs) and solid-state amplifiers are expensive and their cost represents a significant fraction of the project cost. The usage of MW-scale klystrons feeding groups of cavities allows one considerable cost reduction. However such a choice only provides control of the vector sum of the accelerating voltage for the group of cavities, which is usually insufficient for proton or ion accelerators if the beam energy is below about $1 \mathrm{GeV}$ [1]. Therefore RF sources dynamically controlled in phase and power around the carrier frequency and feeding individually each SRF cavity are necessary for large-scale intensity-frontier proton and ion accelerators.

*Corresponding author, Phone: 1-630-340-4911

"e-mail: gkazakevitch@yahoo.com; grigory@muonsinc.com 
Magnetrons are more efficient and less expensive than the above-mentioned traditional RF sources [2]. Thus utilization of magnetron RF sources in large-scale accelerator projects can significantly reduce the RF system cost. As formulated and demonstrated in Ref. [3] magnetron RF sources are suitable for dynamic phase and power control required for stabilization of the accelerating field in SRF cavities. The low cost of magnetron power allows for powering a single cavity which greatly improves the stability of each cavity's voltage and phase.

The magnetron phase is controlled by the phase modulation of the injection-locking signal. The bandwidth of phase control depends on the magnitude of the injection-locking signal and may reach $0.1 \%$ of the magnetron frequency [3]. In particular it is at least $3 \mathrm{MHz}$ for the $2.45 \mathrm{GHz}, 1$ $\mathrm{kW}, \mathrm{CW}$ magnetrons used in our experiments.

Recently two methods of power control in magnetron based transmitters utilizing the wideband phase control were suggested and studied experimentally. In the first method the output power is combined from the outputs of two magnetrons by a 3-dB hybrid combiner [3, 4]. An independent control of both magnetrons phases yields control of their vector sum and, consequently, the phase and power of the output signal at any impedance of the load. The second method suggested in Ref. [5] is suitable for voltage control in high Q-factor cavities only (QLoad $\sim 10^{7}$ or more). In this case the signal which controls the magnetron phase has an additional wideband control of depth of the phase modulation at a frequency much larger than the cavity bandwidth. The cavity voltage control is provided by redistribution of RF power between the fundamental frequency and the sidebands for which power is determined by the depth of phase modulation. Both methods provide practically the same power efficiency of $\sim 50 \%$ at power control in the range of 5-10 dB. Although the second method requires less high power hardware and is less expensive, its usage is limited to sufficiently high Q-factor cavities, because the phase modulation induces a parasitic amplitude modulation of the cavity voltage at harmonics of the phase modulation frequency. The amplitude modulation is increased with reduction of loaded Qfactor.

The two-channel magnetron transmitter with power combining allows a very wide range of power control, certainly sufficient to control the microphonics and beam loading in SRF cavities. However a novel method with a wide-band phase control, but a narrower range of power control at a narrower bandwidth (in the range of a few $\mathrm{kHz}$ ) may be sufficient for many accelerators. The novel concept of magnetron power control based on the wide-range current regulation in an injection-locked magnetron is considered in this paper. The concept is based on proposed and studied the kinetic model of phase focusing in magnetron by the injection-locking signal. The developed method provides the highest efficiency at the given range of power control. Analytical substantiation and experimental verification of the model and concept are also discussed.

\section{Power control in pre-excited magnetrons}

Here we consider how a resonant pre-excitation of magnetrons affects the range of current variation based on a simple model utilizing the drift approximation and perturbation theory. For simplicity, the presented kinetic approach does not consider Larmor's motion of electrons and variations of radius of the Larmor's orbit which is significant at the resonant interaction of the moving charge with a synchronous wave excited in an operating magnetron, [6]. The approach is more acceptable for qualitative consideration of the processes in magnetrons and explanation of our concept than for quantitative calculations, nevertheless it can be used for estimations. We will follow the analytical theory of free running magnetrons developed by P.L. Kapitza [6]. As in 
Ref. [6] we will neglect the beam space charge effects, especially because we consider the CW tubes operating without very high currents.

Let us consider a conventional $N$-cavities magnetron powered somewhat below the critical voltage, with a static constant magnetic field, $H$, above the critical magnetic field. In this consideration, we assume that the magnetron is loaded by a matched load, so that any wave coming to the magnetron is considered to be excited by an external source. In the absence of generation, the motion of electrons in the crossed magnetic and static electric fields is described by a superposition of two circular motions: rotation of the electron at the angular cyclotron (Larmor's) velocity, $\omega=e H /(m c)$ and motion of the centre of this circle around the coordinate frame centre at the angular velocity, so that $\omega>$ [6]. The former motion has a comparatively minor effect on the magnetron operation and can be neglected in the first approximation. The latter motion represents a drift of the transported charge averaged over $\omega$. Under these conditions, the drift equations for a non-generating magnetron in cylindrical coordinates are [7]:

$$
\left\{\begin{array}{l}
\dot{r}=-\frac{c}{H r} \frac{\partial \Phi^{0}}{\partial \varphi}=0 \\
\dot{\varphi}=\frac{c}{H r} \frac{U}{\ln \left(r_{2} / r_{1}\right) r}=\frac{c}{H r} \frac{\partial \Phi^{0}}{\partial r}
\end{array}\right.
$$

Here $\Phi^{0}$ is a potential of the static electric field, so that $E_{r}=\operatorname{grad} \Phi^{0}, \Phi^{0}=U \ln \left(r / r_{1}\right) / \ln \left(r_{2} / r_{1}\right)$, $E_{\varphi}(r)=0, U$ is the magnetron feeding voltage, $r_{l}$ and $r_{2}$ are the magnetron cathode and anode radii, respectively. As one can see from the first equation the radial velocity is equal to zero, and the azimuthal drift is only present, i.e., the magnetron represents a closed diode with a magnetic isolation.

Now we consider the magnetron operating in a nominal regime in the $\pi$-mode. The necessary condition for operation of the magnetron is the presence of a synchronous wave whose frequency, $\omega$, coincides with a harmonic of the charge rotation frequency, i.e., $\omega=n$, where $n$ $=N / 2$. Taking into account that the magnetron operating frequency is much smaller than the frequencies of modes related to the magnetron interaction space $\left(c / r_{1}, c / r_{2}>>\omega / \pi\right)$ the quasistatic approximation can be used. Then, the potential of the rotating wave can be presented in the following well-known form:

$$
\Phi=\sum_{k=-\infty}^{\infty} \frac{\widetilde{E}_{k} \cdot r_{1}}{2 k}\left[\left(\frac{r}{\left(x_{1}\right.}\right)^{k}-\left(\frac{\left(r_{1}\right.}{k}\right)^{k}\right] \sin (k \varphi+\omega t)
$$
where $\tilde{E}_{k}$ is the amplitude of $k$-th harmonic of radial RF electric field at $r=r_{l}$. Note that the form of the potential was chosen so that the azimuthal electric field at the cathode is zero. The coefficients $\tilde{E}_{k}$ are determined by requirement to have zero azimuthal electric field at the anode everywhere except the gaps of the cavities. The term in the sum of Eq. (2) with $k=n$ has a resonant interaction with the azimuthal motion of electrons and therefore makes the major contribution. In the further considerations we will retain only this term.

Adding the potential of the rotating wave into Eqs. (1) due to the perturbation theory one obtains:

$$
\left\{\begin{array}{l}
\dot{r}=-\frac{c}{H r} \frac{\partial}{\partial \varphi}(\Phi) \\
\dot{\varphi}=\frac{c}{H r} \frac{\partial}{\partial r}\left(\Phi^{0}+\Phi\right)
\end{array} .\right.
$$


Rewriting the above equation in the coordinate frame rotating with the synchronous wave (angular frequency of $=\omega / n$ ), and assuming that $\varphi^{\prime}=\varphi+\omega \cdot t / n$ one obtains [7]:

where

$$
\left\{\begin{array}{l}
\dot{r}=-\frac{c}{H r} \frac{\partial}{\partial \varphi^{\prime}} \Phi^{\prime} \\
\dot{\varphi}^{\prime}=\frac{c}{H r} \frac{\partial}{\partial r} \Phi^{\prime}
\end{array}\right.
$$

an effective potential. The first two terms determine the azimuthal drift. They are related to the static electric field and azimuthal drift with angular frequency of the synchronous wave frame, $\omega / n$. The third term determines the azimuthal and radial drifts caused by the RF field of the synchronous wave. Substituting this potential in Eqs. (4) one obtains [7]:

$$
\left\{\begin{array}{l}
\dot{r}=\omega \frac{\breve{r}^{2}}{r} \varepsilon \phi_{1}(r) \cos \left(n \varphi^{\prime}\right) \\
n \dot{\varphi}^{\prime}=-\omega \frac{\breve{r}^{2}}{r}\left(\frac{d \phi_{0}}{d r}+\varepsilon \frac{d \phi_{1}}{d r} \sin \left(n \varphi^{\prime}\right)\right)
\end{array}\right)
$$

Here $\breve{r}=\sqrt{-n c U /\left(\omega H \ln \left(r_{2} / r_{1}\right)\right)}$ is the "synchronous" radius where the drift velocity is equal to the phase velocity of the RF wave $(H<0$ is assumed),

$$
\varepsilon=\widetilde{E}_{n} / E_{1}=\widetilde{E}_{n} \cdot r_{1} \ln \left(r_{2} / r_{1}\right) / U, \phi_{0}(r)=\ln \frac{r}{r_{1}}-\frac{1}{2}\left(\frac{r}{k}\right)^{2}, \phi_{1}(r)=\frac{1}{2 n}\left[\left(\frac{r}{x_{1}}\right)^{n}-\left(\frac{(}{r}\right)^{n}\right] .
$$

The first equation in Eqs. (5) describes the radial velocity of the moving charge in the rotating frame of the synchronous wave. In accordance with this equation, the drift of charge towards the anode is possible at $-\pi / 2<n \varphi^{\prime}<\pi / 2$ with a period of $2 \pi$, i.e., only in "spokes". The charge can enter the spoke through the boundaries located at $\pm \pi / 2$. The radial drift velocity is proportional to $\varepsilon$. The condition $\varepsilon \geq 1$ does not allow functioning of magnetron.

The second equation represents the azimuthal velocity of the drifting charge in the frame of synchronous wave. The second term in the brackets causes the resonant RF focusing of the charge. The first term of the equation describes a radially-dependent azimuthal drift of charge caused by the rotating frame with azimuthal velocity $-\omega / n$. This results in an additional nonresonant azimuthal drift of the charge dependent on radius and in a migration of charge from a "spoke" at low $\varepsilon$, i.e., in a defocusing of the charge. At the "synchronous" radius the defocusing is absent since $d \phi_{0} / d r=0$.

Analysis of Eqs. (5) shows that at low $\varepsilon$ the stationary points appear where the radial and azimuthal velocities are zero. Consequently, the point locations are determined by following equations: $\dot{r}=0, n \dot{\varphi}^{\prime}=0$. The solution results in the points being located on the boundaries of the azimuthal space allowed for a "spoke" $(-\pi / 2, \pi / 2)$ and are determined by the equations: $n \varphi^{\prime}= \pm \pi / 2$, and $d \phi_{0} / d r= \pm \varepsilon \cdot d \phi_{1} / d r$. The stationary points do not allow drift of the charge towards the magnetron anode thus stopping the resonant focusing. Since motion of charge drifting to the magnetron anode is possible only in "spokes", the absence of the azimuthal focusing stops coherent oscillation in the magnetron. 
The Eqs. (5) were numerically integrated at various $\varepsilon$ for a typical magnetron model, [7], where $N=8, r_{1}=5 \mathrm{~mm}, r_{2} / r_{1}=1.5, \breve{r} / r_{1}=1.2$, beginning from $r_{m i n}=a+r_{1}$, where $a$ is the Larmor's radius of electron nearby the cathode. Since we consider coherent generation of the charge delivered to the magnetron anode we analyse behaviour of the squared charge delivered due to the drift.

Analysis of the charge trajectories vs. radius at $\varepsilon<0.3$ shows migration of the charge from the azimuthal interval $( \pm \pi / 2)$ allowed for a "spoke" because of the defocusing term in Eqs. (5). Out of the $( \pm \pi / 2)$ interval the focusing is absent and the emigrated electrons absorb the RF energy from the synchronous wave. This increases Larmor's radius of the electrons and returns the electrons to the cathode. The residual squared charge delivered to the magnetron anode is plotted in Fig. 1, curve 1. However in this curve we did not consider losses of electrons at low $\varepsilon$ and violation of synchronism at long time of the drift, while it is important for coherent oscillation in magnetron. Thus we corrected the squared charge by the limitation of the charge drift time estimated as $\leq 10$ cyclotron periods for a typical magnetron, [7]. The corrected plot is shown in Fig. 1 by curve 2 . We also considered the drift time of charge no shorter than 2-3 cyclotron periods analysing computed electron trajectories shown in Ref. [8]; the shorter time is insufficient to form "spokes". The resulted corrections are plotted in Fig. 1 by curve 3. The both limitations of the drift time one can consider as conditions for applicability of the Eqs. (5).

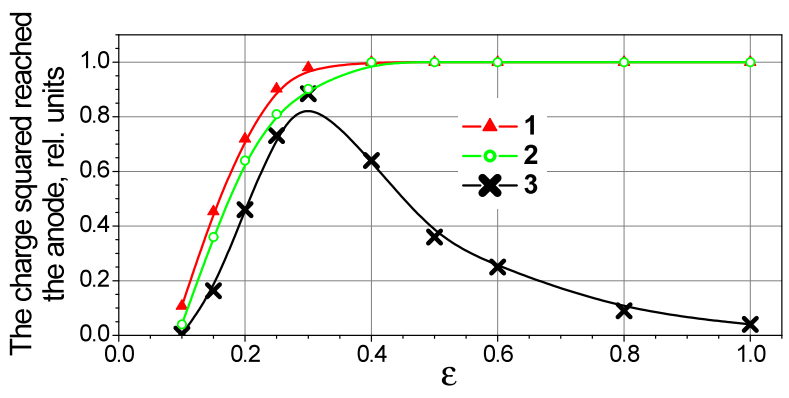

Fig. 1. Behaviour of the squared charge delivered to anode in a typical magnetron model vs. $\varepsilon$.

One can assume that the maximum of the curve 3, Fig. 1, determines amplitude of the RF field in the synchronous wave necessary for self-excitation of magnetron if $U$ is the critical (Hartree) voltage. An increase of the synchronous wave amplitude affects the allowable charge delivered to the magnetron anode, i.e., the magnetron current, formally as a decrease of $U$. Thus one can assume that driving of the magnetron by a resonant wave with power $P_{D}$, comparable to the power in the synchronous wave, $P_{S}$, (the both waves are in phase in a steady state) will provide start-up and stable operation of the magnetron even if the magnetron feeding voltage is somewhat less than the threshold of self-excitation. This will provide stable operation of the magnetron with current less than the minimum current in free run, i.e., in the extended range of regulation of the magnetron current (power). It is the basis of our concept. As it follows from performance charts, the minimum magnetron power, $P_{\min }$, is usually $\sim 1 / 3$ of the nominal power, $P_{n o m}$, and corresponds to the threshold voltage. For commercial magnetrons, as it is noted in Ref. [6], the energy of the RF field in the interaction space is approximately in 3 times less than the energy stored in the magnetron RF system including the interaction space and cavities; half of the energy in interaction space is associated with the RF field in the synchronous wave. Thus pre-exciting of the magnetrons by the resonant driving wave with power $P_{D}$ is equivalent to decrease of $U$ by $U \sim U \cdot\left(1-\left(P_{S \min } /\left(P_{S \min }+P_{D} / 6\right)\right)^{1 / 2}\right)$. Then the minimum current, $I_{m i n}$, of the 
driven magnetron with the dynamic impedance $Z_{D}=U / I$ one estimates as: $I_{\min D} \sim I_{\min }-U / Z_{D}$, where $I_{\min }$ is the minimum current in free run. The power range, $R_{D}$, regulated in the driven magnetron one estimates at the nominal magnetron current, $I_{\text {nom }}$, as: $R_{D^{\sim}}\left(I_{\text {nom }} / I_{\text {munD }}\right)$. For the magnetron model at $P_{D^{\sim}}-10 \mathrm{~dB}$ of the nominal power one obtains: $R_{D^{\sim}} 10 \mathrm{~dB}$. As it is shown below, the estimates correspond to measured results.

In the case of pre-excitation the magnetron power is determined by the magnetron current at a given power of the synchronous wave. A lack of RF voltage in the synchronous wave at the lower magnetron current is compensated by the injection-locking signal. Thus, for a magnetron pre-excited by the injection-locking signal, the threshold voltage of coherent generation depends on the magnitude of pre-excitation. Therefore the injection-locked magnetron at a given magnetic field is described by a family of Volt-Amp (V-I) characteristics depending on values of the pre-exciting signal. The characteristics determine the value of $I_{\min D}$ at the given feeding voltage and the power of the pre-exciting injection-locking signal.

Thus the resonant pre-excitation of magnetrons extends the range where their power can be regulated. The regulation can be achieved by control of current in a high voltage power supply managed with current stabilization feedback. In the case of a switching power supply the bandwidth of current control depends on the switching frequency and may reach $\sim 10 \mathrm{kHz}$ without compromising power efficiency.

Since the process of coherent oscillation in magnetrons is highly efficient, one can expect conservation of high efficiency in the proposed extended range of power control.

\section{Experimental verification of the power control in pre-excited magnetrons}

The described kinetic model and the concept of the power variation in pre-excited magnetrons were verified in experiments with $1 \mathrm{~kW}, 2.45 \mathrm{GHz}$ tubes operating in pulsed and $\mathrm{CW}$ regimes. The OM75P(31) magnetron with a permanent magnet was used for measurements in the pulsed regime. It was powered by a pulsed modulator with partial discharge of a $200 \mathrm{~F}$ storage capacitor, [9]. This magnetron had been used already for 8 years and therefore could provide only about half its nominal output power because of "tired" cathode. A schematic of experimental setup is shown in Figs. 2, 3, [3].

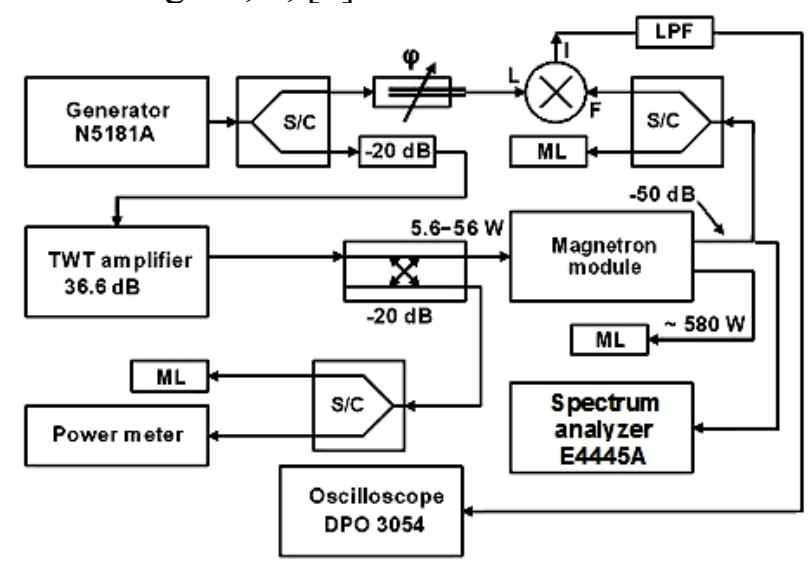

Fig. 2. Schematic of experimental setup to measure the magnetron current, voltage, spectrum, output power and power of the pre-exciting signal at the power variation. S/C denotes a 3-dB splitter/combiner, ML is a matched load. 


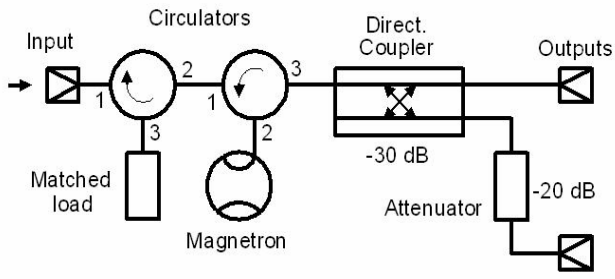

Fig. 3. Scheme of the magnetron module.

The pulse duration for the measurements was chosen to be $1.2 \mathrm{~ms}$. The level of voltage ripple at the magnetron is negligible in the modulator, however, the capacitor introduces a voltage droop $<0.15 \%$ during a $1.2 \mathrm{~ms}$ pulse. The magnetron modulator was fed by a switching High Voltage (HV) stabilized power supply.

The magnetron current and pulsed cathode voltage were measured by a calibrated transducer installed in the magnetron cathode circuitry and by a calibrated HV compensated divider, respectively. Inaccuracy of the measurements was less than $\pm 1 \%$.

The measurements were conducted with the magnetron operating in free running mode and with the pre-excited magnetron. In the latter case the magnetron was frequency-locked by the CW TWT amplifier driven by CW Agilent generator N5181A as shown in Fig. 2. The magnetron carrier frequency and pulsed power were measured by the E4445A spectrum analyzer. The setup allowed the measurement of the phase pushing vs. current of the magnetron by a phase detector at various power levels of the locking signal. The locking frequency offset of $-2.8 \mathrm{MHz}$ relative to the magnetron average free run frequency at an output power of $\approx 450 \mathrm{~W}$ was used.

The traces of the magnetron voltage vs. locking power were measured at the minimum possible voltage of the operating magnetron, and at the voltage when the magnetron power was $450 \pm 8$ W, Fig. 4.

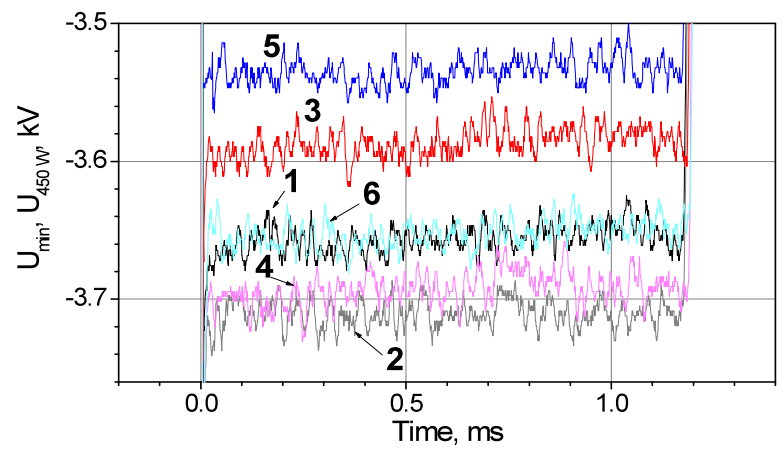

Fig. 4. Traces of the magnetron voltage vs. locking power.

In Fig. 4 is plotted measured pulsed magnetron voltage at the minimum generated power, traces 1, 3, 5, and at a power of $450 \pm 8 \mathrm{~W}$, traces 2, 4, 6 at the following powers of the frequency-locking signal, $\mathrm{P}_{\text {Lock: }} 12 \mathrm{~W}$, traces 1 and $2 ; 27.4 \mathrm{~W}$, traces 3 and $4 ; 53.9 \mathrm{~W}$, traces 5 and 6 , respectively.

The plots clearly demonstrate a decrease of the magnetron start up voltage modulus, $|\mathrm{U}|$, at an increase of power of the locking signal as discussed above. The start up voltage modulus, $|\mathrm{U}|$, in accordance with the locking power is notably less than the modulus of the magnetron critical voltage, $|3,737 \pm 9 \mathrm{~V}|$, measured at free run. The increase of the locking signal also decreases the magnetron voltage necessary for generation of the required power. The decrease of the 
magnetron start-up voltage vs. power of the magnetron pre-exciting signal, $\mathrm{P}_{\text {Lock }}$, is plotted in Fig. 5. The point $P_{\text {Lock }}=0$ is related to the free run critical voltage.

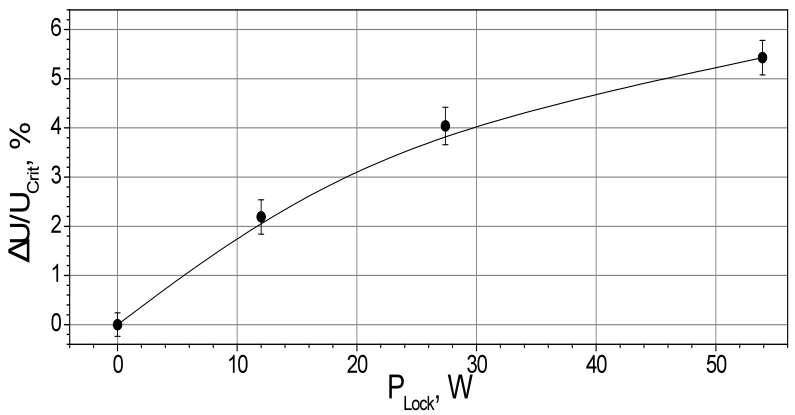

Fig. 5. Lowering of magnetron start-up voltage in percents vs. power $\left(\mathrm{P}_{\text {Lock }}\right)$ of the signal, preexciting the magnetron. The solid line shows B-spline fit of the measured data, error bars show the rms errors.

Measured traces of pulses of the minimum possible magnetron current and the current at a magnetron power of $450 \pm 8 \mathrm{~W}$ for various locking powers are shown in Fig. 6.

The plots in Figs 4-6 demonstrate Proof-of-Principle of the concept of an increase of the allowed range of the magnetron current at the pre-exciting signal when the magnetron voltage is less than the critical one, measured at free run. Therefore the required range of the magnetron power control can be achieved by variation of the magnetron current (in this measurement varying the magnetron voltage in a narrow range) at the given values of the pre-exciting signal and using the magnetron feeding voltage less than the critical voltage in free run.

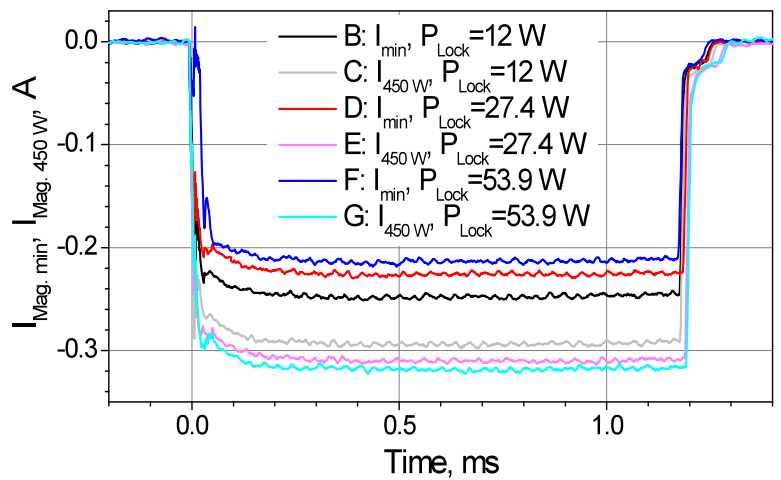

Fig. 6. Traces of the measured pulsed cathode current at minimum generated power and at a power of $450 \pm 8 \mathrm{~W}$ at various powers of the frequency-locking signal, $\mathrm{P}_{\text {Lock. }}$

The plots shown in Fig. 7 demonstrate proportionality of the magnetron power to the magnetron current. The reachable range of the power variation demonstrates a notable increase for the pre-excited injection-locked magnetron in comparison with the free running one when $\mathrm{P}_{\text {Lock }}=0$. 


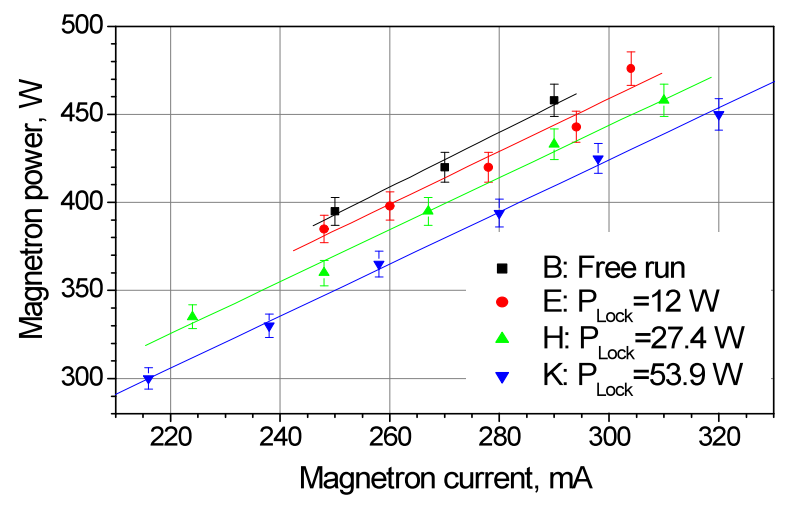

Fig. 7. Dots with error bars: dependence of the magnetron pulsed power on the magnetron current for free running and pre-excited tube at various power of the pre-exciting signal. Solid lines: linear fits of the measured data.

Measured V-I characteristics of the magnetron at free run, and also being pre-excited, injectionlocked, are plotted in Fig. 8.

The fits plotted in this figure show that the dynamic impedance, $Z_{D}$, of the operating magnetron remains constant, to good accuracy, at various values of the magnetron current. Since the magnetron power, $P$, in the first approximation is proportional to the magnetron current, $I$, one can write: $U / U \sim\left(Z_{D} / Z_{S}\right) \cdot(P / P)$. Here $Z_{S}=U / I$ is the magnetron static impedance. In magnetrons $Z_{D} / Z_{S} \sim 0.1$, thus one can see that for quite deep variation of the magnetron current (power) one needs much smaller variation of the magnetron voltage at the respective magnitude of the injection-locking signal.

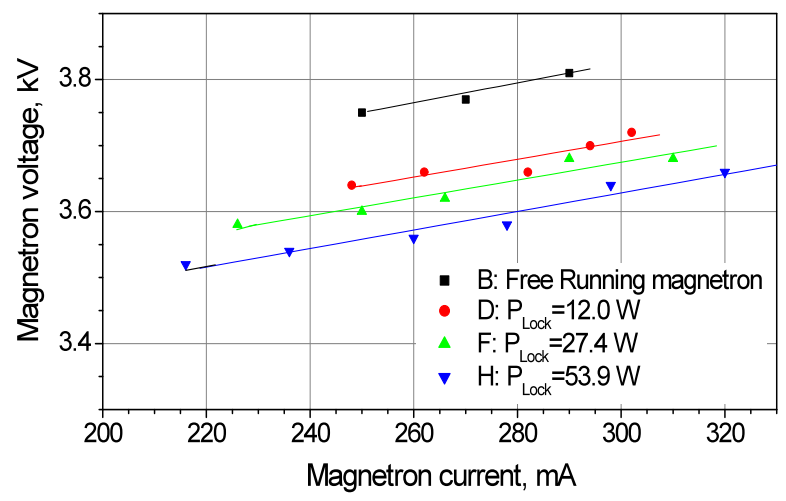

295

296

297

298

299

300

301

302
Fig. 8. V-I characteristics of the free running, dots B, or pre-excited injection-locked magnetron, dots D, F, H at constant magnetic field. Solid lines are linear fits.

Note, that at all the measurements with various power of the driving signal and various power of the magnetron the tube was frequency-locked.

More detailed measurements of power regulation at various values of the pre-exciting locking power were performed in CW mode with $2.45 \mathrm{GHz} 1.2 \mathrm{~kW}$ magnetron type YJ1540 having a permanent magnet. A schematic of the measurements is shown in Fig. 9. 


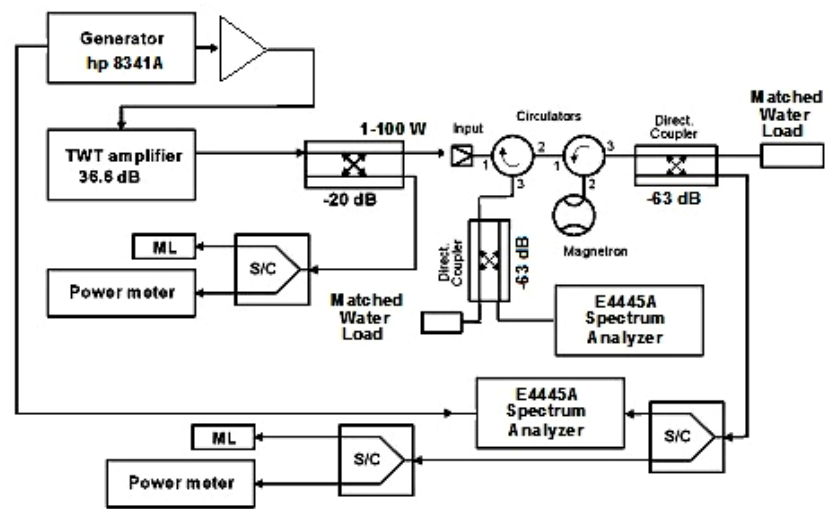

Fig. 9. Schematic of the magnetron CW setup to test the power control.

The magnetron was frequency-locked by the HP 8341A generator via a Solid-State Amplifier (SSA) and $36.6 \mathrm{~dB}$ TWT amplifier providing the CW locking power up to $100 \mathrm{~W}$. The magnetron was powered by an Alter switching high voltage power supply type SM445G, operating as a current source allowing current control.

The proposed method of power control in the injection-locked magnetron, as it was discussed above, utilizes contributions of the locking signal and the magnetron current into the RF field of the synchronous wave. Therefore, to compensate insufficient contribution of a low magnetron current into the RF field, a sufficient value of the locking power is required. The range of the power control depends on the locking power. At the insufficient power, in accordance with Eqs. (5), the defocusing term worsens the phase focusing of the charge at various radii of the trajectory. This increases phase oscillations of the drifting charge contributing to the magnetron phase noise. The measured ratio of power of the carrier frequency to noise in the $\pm 15 \mathrm{MHz}$ offset vs. power of the locking signal, Fig. 10, demonstrates the capability of wide range of power control of the injection-locked magnetron with low noise at a sufficient locking signal.

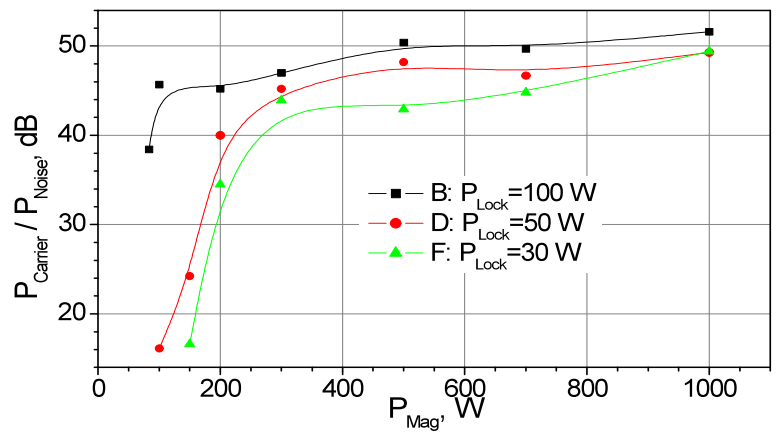

Fig. 10. Measured ratio of power at the carrier frequency to power of noise at various powers of the locking signal in dependence of the magnetron power.

The plots demonstrate the capability of the magnetron to vary power in the range of $10 \mathrm{~dB}(0.1$ $\mathrm{kW}-1 \mathrm{~kW}$ ) at low noise, when the power of the locking signal is $100 \mathrm{~W}$.

The measured spectral density of noise of the injection-locked magnetrons in the range of output power of $10 \mathrm{~dB}$ did not demonstrate a notable increase at low power of the magnetron at $\mathrm{P}_{\text {Lock }}=100 \mathrm{~W}(-10.8 \mathrm{~dB})$, Fig. 11. Note that all the measurements were carried out at the nominal magnetron filament power. 


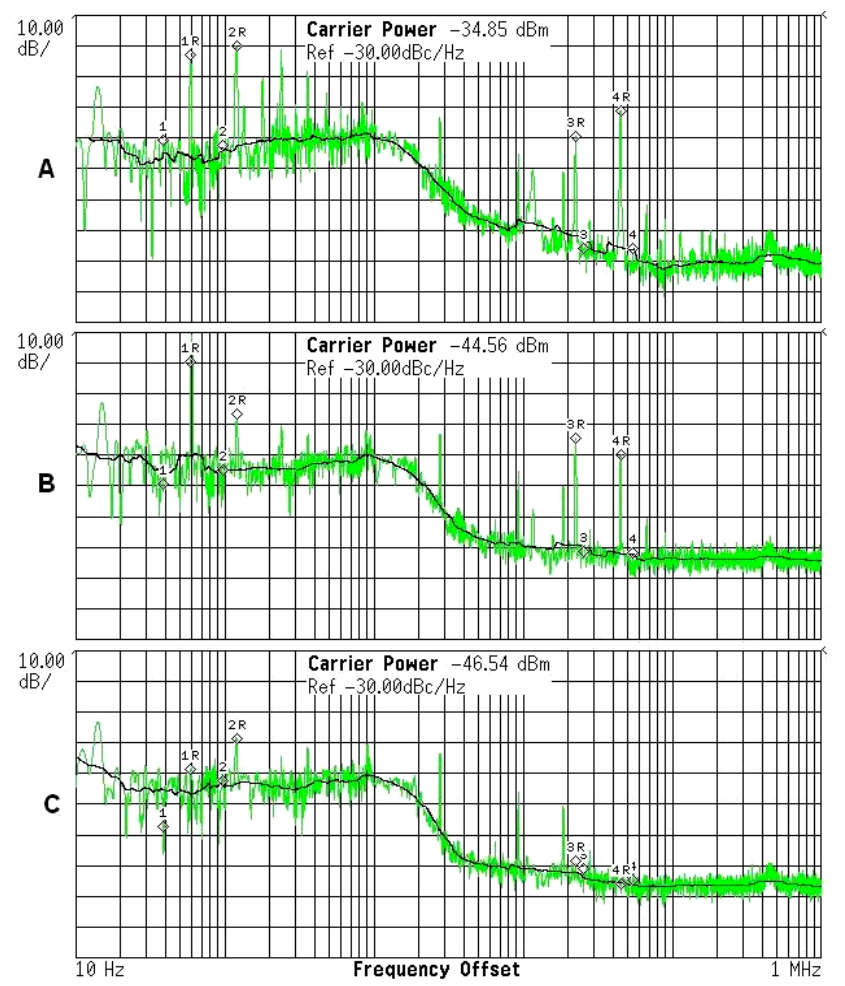

Fig. 11. Spectral density of noise of the frequency-locked magnetron at output power of $1000 \mathrm{~W}$ and $100 \mathrm{~W}$, traces $\mathrm{A}$ and $\mathrm{B}$, respectively. Traces $\mathrm{C}$ are the spectral density of the injectionlocking signal. The black traces show the averaged spectral density of noise.

Trace $\mathrm{A}$ is the spectral density at $\mathrm{P}_{\mathrm{Mag}}=1000 \mathrm{~W}, \mathrm{P}_{\text {Lock }}=100 \mathrm{~W}$, trace $\mathrm{B}$ is the spectral density at $\mathrm{P}_{\text {Mag }}=100 \mathrm{~W}, \mathrm{P}_{\text {Lock }}=100 \mathrm{~W}$. The trace was measured at operation of the magnetron with a voltage less than critical in free run. Trace $\mathrm{C}$ is the spectral density of noise of the magnetron preexciting signal with a power level of $100 \mathrm{~W}$, when the magnetron high voltage was OFF.

The trace $\mathrm{C}$ shows sidebands of the injection-locking signal caused by switching power supplies of the TWT amplifier. Other sidebands shown in traces A and B are associated with the SM445G switching power supply.

Comparison of traces $\mathrm{A}$ and $\mathrm{B}$ shows that the noise in both traces is approximately the same, i.e., in all the range of power control the phase focusing of the charge in "spokes" is sufficient, therefore the regime at $\mathrm{P}_{\text {Lock }}=-10.8 \mathrm{~dB}$ at such power control in the range of $10 \mathrm{~dB}$ is stable with low phase noise. This verifies the adequacy of the proposed method of power control by the preexcited injection-locked magnetron fed by a voltage less than the critical voltage in free run for powering SRF cavities.

A decrease of the locking power by $3 \mathrm{~dB}$ or more increases quite notably the noise at low power of the magnetron in the range of $1 \mathrm{kHz}-1 \mathrm{MHz}$, Fig. 12. 


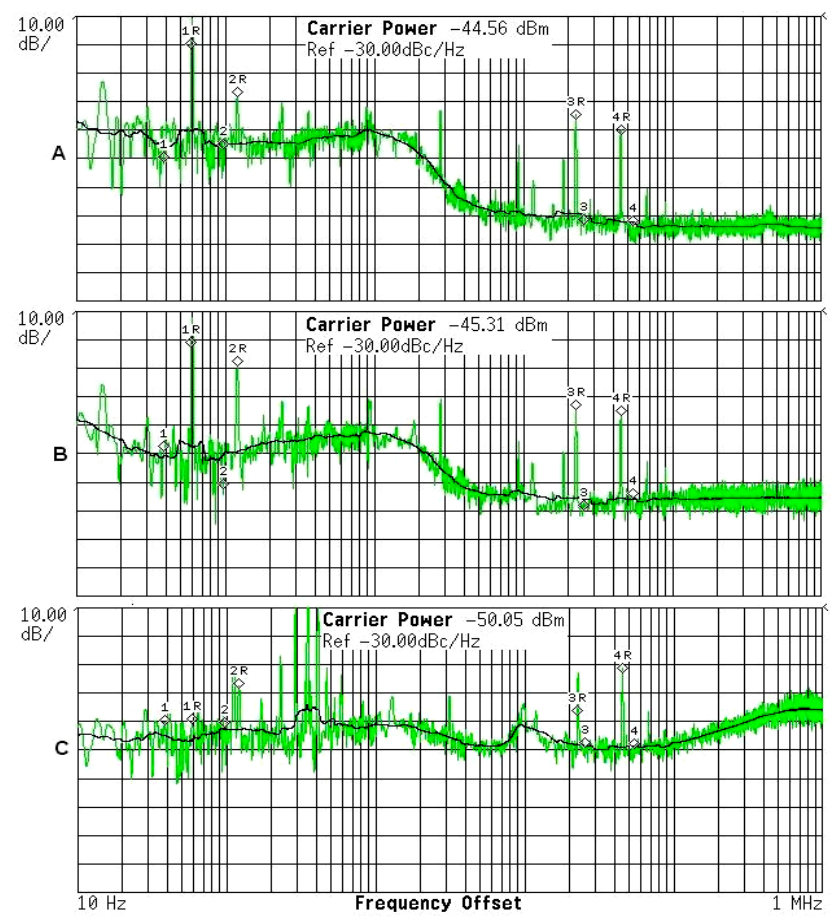

Fig. 12. Spectral density of noise at various power levels of the locking signal at $\mathrm{P}_{\mathrm{Mag}}=100 \mathrm{~W}$. Trace A $-\mathrm{P}_{\text {Lock }}=100 \mathrm{~W}$, trace $\mathrm{B}-\mathrm{P}_{\text {Lock }}=50 \mathrm{~W}$, trace $\mathrm{C}-\mathrm{P}_{\text {Lock }}=30 \mathrm{~W}$. Black traces are the averaged spectral density of noise.

It means that the phase focusing at $\mathrm{P}_{\text {Lock }} 50 \mathrm{~W}(-13.8 \mathrm{~dB}$ of the max. power for a $1.2 \mathrm{~kW}$ tube) and $\mathrm{P}_{\mathrm{Mag}}=100 \mathrm{~W}$ is insufficient. This increases the magnitude of the azimuthal oscillations of the charge in the "spokes", increasing the phase noise at low magnetron power while the magnetron remains frequency-locked, Fig. 13.

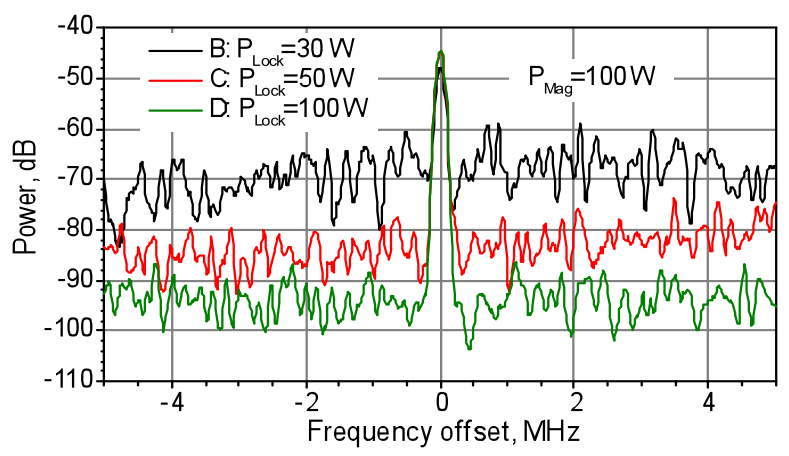

Fig. 13. Spectral distributions of noise in a neighbourhood of the carrier frequency vs. $\mathrm{P}_{\text {Lock }}$ at $\mathrm{P}_{\mathrm{Mag}}=100 \mathrm{~W}$.

Note that the azimuthal oscillations of charge in the "spokes" in the pre-excited injectionlocked magnetron decrease the stability of the tube and may stop operation of the magnetron at an additional decrease of the magnetron current or the magnitude (power) of the pre-exciting injection-locking signal.

The efficiency of the controlled magnetron, $\eta$, measured in the total range of power control remains highest, Figs. 14, 15 in comparison with other methods of power control in transmitters with frequency-locked magnetrons, $[3,5]$. 


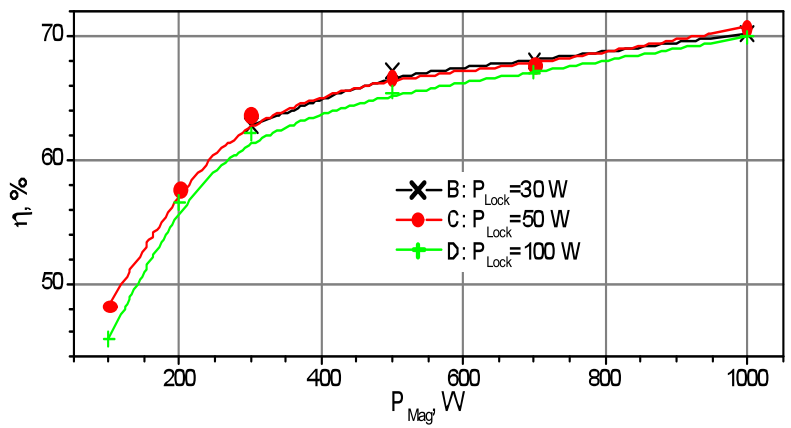

Fig. 14. Measured absolute efficiency of the injection-locked $1.2 \mathrm{~kW}$ magnetron with power control by management of the magnetron current in a wide range.

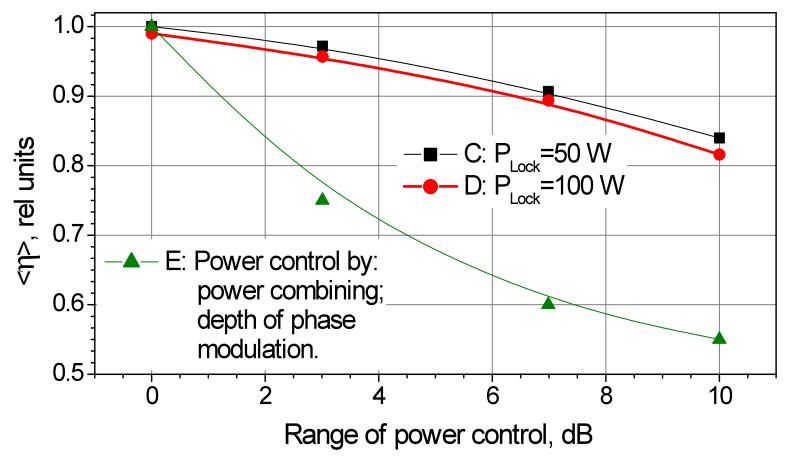

Fig. 15. Relative magnetron efficiency vs. range of power variations at various methods of control. Traces $\mathrm{C}$ and $\mathrm{D}$ are average efficiency of the pre-excited injection-locked $1.2 \mathrm{~kW}$ magnetron measured at the extended current control.

For comparison in Fig. 15 is plotted trace E presenting calculated average efficiency of $1 \mathrm{~kW}$ magnetrons at power combining, [3], or at management of the depth of phase modulation, [5].

Spectra of the carrier frequency of the injection-locked magnetron at various power levels in the range of $10 \mathrm{~dB}$ by the proposed power control are precisely-stable and do not demonstrate any broadening or shifts, Fig. 16. This verifies the adequacy of the proposed power control in the injection-locked magnetrons to requirements of the SRF cavities.

Shown in the plots, trace $\mathrm{P}_{\mathrm{Mag}}=0.0 \mathrm{~W}, \mathrm{P}_{\mathrm{Lock}}=30 \mathrm{~W}$ corresponds to measurements with magnetron anode voltage turned OFF. It represents the injection-locking signal.

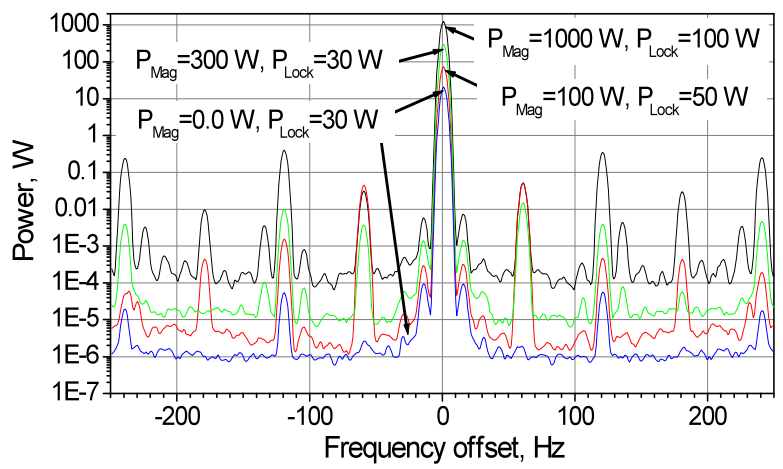

Fig. 16. Offset of the carrier frequency at various power levels of the magnetron, $\mathrm{P}_{\text {Mag }}$, and the locking signal, $\mathrm{P}_{\text {Lock }}$. 
Results of measurements shown in Figs 5-8 and 10-16 demonstrate Proof-of-Principle of power control in frequency-locked magnetrons by extended variation of the magnetron current and substantiate the proposed method. The method provides the necessary range of the power control in magnetron RF sources intended for the feeding of SRF cavities in multi-experiment accelerators if the voltage of the magnetron power supply may be less than the critical voltage in free run.

Note, that the measured dependence of the magnetron $\mathrm{CW}$ power on the magnetron current is linear in the full range of the control, Fig. 17.

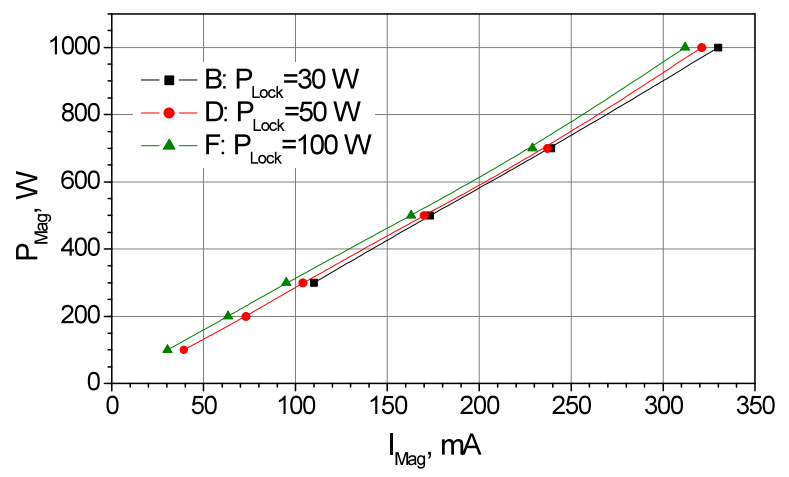

Fig. 17. Measured dependence of power of the injection-locked magnetron on the controlled CW magnetron current.

The bandwidth of the phase control of the injection-locked magnetron is determined by duration of the transient process at the phase control. The duration is determined by time of establishment of the forced oscillation in the synchronous wave, providing phase focusing, and in the magnetron cavity. Duration of the transient process in the magnetron cavity is determined by the cavity filling time and in first approximation does not depend on the amplitude of oscillation in the cavity, i.e., on the magnetron power. The relatively large power of the injection-locking signal required for the power control at the extended range of the magnetron current increases radial drift velocity of charge. This does not prolong the transient process establishing forced oscillations in the synchronous wave. Therefore one can expect that the bandwidth of the phase control of injection-locked magnetrons operating at a voltage less than critical in free run (if the magnitude of the synchronous wave is sufficient for the required phase focusing) can not be significantly narrower at a sufficient locking signal than the bandwidth of the tube in the nominal regime. The similarity of spectra of the sidebands resulting from powering of the injection-locked magnetron and the TWT amplifier by the switching power supplies at a wide range of power control (100-1000 W), and the spectral distributions of noise up to $1 \mathrm{MHz}$, Fig. 11, indicate that operation of the magnetrons below the critical voltage at $\mathrm{P}_{\text {Lock }} \approx-10 \mathrm{~dB}$ does not limit the bandwidth of the phase control in first approximation.

The bandwidth of the proposed power control in the case of utilizing a switching power supply is determined by the switching frequency of the HV power supply, which usually is $\sim 20 \mathrm{kHz}$. An increase of the switching frequency will allow a bandwidth of the power control up to a few $\mathrm{kHz}$. In this case one can expect suppression of the mechanical noises (microphonics, etc.) by $\sim 40 \mathrm{~dB}$ or more.

Experiments with two 2.45, GHz, $1 \mathrm{~kW}, \mathrm{CW}$ magnetrons having in free run mode a frequency difference of about 5.7 MHz and frequency-locked at the same (middle) frequency, demonstrated in the 2-cascade magnetron setup a bandwidth of the phase control of a few $\mathrm{MHz}$ at a power of the injection-locking signal of $-25 \mathrm{~dB}$, [3]. It is a basis of a single-channel, high-power, highly 
efficient, cost-effective, 2-cascade magnetron transmitter concept in which the high power magnetron may operate at the voltage below the threshold of self-excitation.

Therefore utilization of the proposed method of power control in frequency-locked magnetrons simplifies the conceptual scheme of the magnetron transmitter allowing mid-frequency power control in the range of $10 \mathrm{~dB}$ at a wide-band phase control. A single-channel magnetron transmitter can be used as such a high-power RF source, like as in the method described in Ref. 5 , however our method provides highest efficiency and it is applicable for any load impedance.

The control (modulation) of the magnetron current causes phase pushing in the frequencylocked magnetron, thus elimination of the parasitic phase modulation, caused by the phase pushing in the high-power magnetron is very important. At a bandwidth of the phase control in the $\mathrm{MHz}$ range, one can expect the phase pushing elimination to a level of about $-50 \mathrm{~dB}$ or less, suitable for various superconducting accelerators. Note that the increase of the injection-locking signal to a level of about $-10 \mathrm{~dB}$ notably decreases the phase pushing as it was shown in Ref. [3].

As it was demonstrated above, the proposed method of power control in magnetrons provides the highest efficiency of the transmitter.

Capability of the injection-locked magnetron for a deep dynamic current (power) control was experimentally verified using a harmonic modulation of the magnetron current by an external voltage controlling the power supply. Traces of harmonic modulation of RF power by the injection-locked magnetron fed by switching power supply type SM445G are shown in Fig. 18.

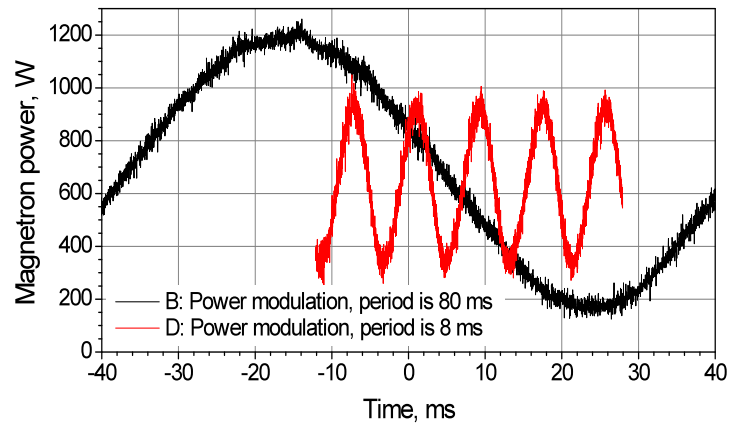

Fig. 18. Modulation of the magnetron RF power performed controlling the magnetron current by the high-voltage switching power supply at $\mathrm{P}_{\text {Lock }}=100 \mathrm{~W}$.

The plots were obtained controlling the power supply by harmonic voltages with periods of 80 $\mathrm{ms}$ and $8 \mathrm{~ms}$. The traces representing harmonic modulations of the RF power were measured as modulation of the magnetron current calibrated in RF power with an accuracy of about $1 \%$ and averaged over 16 runs because of high noise caused by the switching power supply. Note that the used power supply was not intended for power modulation; it has insufficient bandwidth of the feedback loop limiting the bandwidth of the magnetron current (power) control.

As it was demonstrated and discussed in the presented work and in Ref. [3] for the magnetrons excited by a relatively large (about of $-10 \mathrm{~dB}$ ) injection-locking signal the methods of wide range mid-frequency power control and wide-band phase control, and their combination as well, look feasible. Thus the magnetron transmitters utilizing injection-locking signal with the relatively large power for operation below the voltage of self-excitation of magnetrons are promising as cost effective, highly-efficient high power RF sources for large projects of superconducting accelerators.

\section{Summary}


A kinetic model considering phase focusing in magnetrons by an injection-locking signal was proposed and studied, The kinetic approach based on the charge drift approximation substantiating stable operation of the pre-excited injection-locked magnetrons below the threshold voltage of self-excitation was proposed and analysed within the framework of the model. A performed analysis of the drift equations allowed for estimating the power of the preexciting resonant signal sufficient for stimulated coherent oscillations in the magnetron at a voltage less than critical in free run. The analysis and experiments demonstrates the capability of magnetrons for wide range power control (up to $10 \mathrm{~dB}$ ) at the sufficient value (about of $-10 \mathrm{~dB}$ ) of the pre-exciting resonant signal.

A novel method of mid-frequency power control of frequency-locked magnetrons, based on analysis of kinetics of the drifting charge has been proposed, analyzed and verified in experiments with $2.45 \mathrm{GHz}, 1 \mathrm{~kW}$ magnetrons. The method was realized by a pre-excitation of the magnetron with a sufficient injection-locking signal. It allows operation of the pre-excited injection-locked magnetron at a voltage less than the critical voltage in free run. This provides variation of the magnetron power in the range up to $10 \mathrm{~dB}$ at precisely-stable carrier frequency and low phase noise that is important for various superconducting accelerator projects. The method allows a mid-frequency dynamic management of the current (power) of the transmitter based on injection-locked magnetrons in the range required for the SRF cavities of multi-task projects keeping capabilities for a wide-band phase control. The proposed method of power control provides the highest efficiency. The bandwidth of power control of the proposed method utilizing a switching HV power supply operating as a current source is determined by the switching frequency. Presently the bandwidth can reach a few $\mathrm{kHz}$.

\section{Acknowledgement}

This work was supported by Fermi Research Alliance, LLC under Contract No. De-AC0207CH11359 with the US DOE.

We are very thankful to Dr. Helen Edwards, for permanent interest and support of this work, Dr. Stephen Holmes and Prof. Nigel Lockyer for the opportunity to carry out these experiments, Mr. Brian Chase and Mr. Ralph Pasquinelli for help in experiments and Dr. R. Thurman-Keup for fruitful discussions.

\section{References}

[1] Project X Reference Design Document: http://projectx-docdb.fnal.gov/cgi-bin/ShowDocument?docid=776

[2] G. Kazakevich, "High-Power Magnetron RF Source for Intensity-Frontier Superconducting Linacs”, EIC 2014, TUDF1132_TALK, http://appora.fnal.gov/pls/eic14/agenda.full

[3] G. Kazakevich, R. Johnson, G. Flanagan, F. Marhauser, V. Yakovlev, B. Chase, V. Lebedev, S. Nagaitsev, R. Pasquinelli, N. Solyak, K. Quinn, D. Wolff, and V. Pavlov, Nucl. Instr. \& Methods in Physics Research A 760 (2014) 19-27.

[4] G. Kazakevich, R. Johnson, B. Chase, R. Pasquinelli, V. Yakovlev, "Regime of a wideband phase-amplitude modulation in a CW magnetron transmitter with a phase control", http://arxiv.org/abs/1407.0304

[5] B. Chase, R. Pasquinelli, E. Cullerton, Ph. Varghese, JINST, 10, P03007, 2015.

[6] P.L. Kapitza, HIGH POWER ELECTRONICS, Sov. Phys. Uspekhi, V 5, \# 5, 777-826, 1963. 
505

506

507

508

509

510

[7] L.A. Vanstein and V.A. Soltsev, in "Lectures on microwave electronics", Moscow, Sov. Radio, 1973.

[8] G.B. Collins, from "Microwave Magnetrons", New York, McGraw-Hill Book Co., 1948.

[9] G. Kazakevich, "Application of magnetrons for intensity-frontier superconducting linacs", http://beamdocs.fnal.gov/ADpublic/DocDB/ShowDocument?docid=4445 